\title{
Cost-Utility Analysis of Antibiotics Compared with Operative Treatment in Uncomplicated Acute Appendicitis.
}

\author{
Napaphat Poprom \\ Mahidol University \\ Oraluck Pattanaprateep ( $\nabla$ oraluck.pat@mahidol.edu ) \\ Mahidol University \\ Chumpon Wilasrusmee \\ Mahidol University \\ Sasivimol Rattanasiri \\ Mahidol University \\ Gareth McKay \\ Queen's University of Belfast, Belfast, Northern Ireland BT12 6BA \\ John Attia \\ University of Newcastle \\ Ammarin Thakkinstian \\ Mahidol University
}

\section{Research Article}

Keywords: cost-utility, antibiotics, acute appendicitis, ICERs

Posted Date: September 16th, 2021

DOl: https://doi.org/10.21203/rs.3.rs-857893/v1

License: (1) This work is licensed under a Creative Commons Attribution 4.0 International License. Read Full License 


\section{Abstract}

Background: Appendicitis is the most common acute abdominal condition affecting general surgical practice. Although appendectomy has been considered the "gold standard" treatment, it has been associated with post-operative complications. Recently, conservative treatment with antibiotics has been considered as an alternative. Therefore, this study was conducted to evaluate if antibiotics could be costeffective when compared to laparoscopic appendectomy (LA) or open appendectomy (OA).

Methods: A prospective study was undertaken to estimate health-related quality of life using a European Quality of Life-5 Dimensions questionnaire for antibiotic and operative treatment and to ascertain indirect and direct non-medical costs in a cohort between November 2018 and October 2019. An incremental costeffectiveness ratio (ICER) at 1 month and a net benefit at 1 year after surgery were estimated. A Tornado diagram was plotted to determine one-way and probabilistic sensitivity analyses; a cost-effectiveness (CE) scatter plot and CE acceptability curve were also generated.

Results: ICERs were estimated from cost-utility analysis comparisons between individual antibiotics (betalactam, quinolone, and cephalosporin + metronidazole), LA, and OA in uncomplicated acute appendicitis. Antibiotics showed improved cost utility compared to operative treatments with an ICER of $-9,585.66$ USD per utility gained at 1 month, and the net benefit at 1 year was 3,250.08 USD.

Conclusions: Base on short term evaluation, antibiotics might represent a lower cost treatment option with better cost utility compared to operative treatment options in uncomplicated acute appendicitis patients. As such, antibiotic treatment might be considered as an alternative option where resources are limited to minimize complication rates associated with operative treatments.

\section{Introduction}

Appendicitis is the most common acute abdominal condition in general surgical practice with an approximate incidence of 1 in 1000 person years ${ }^{1,2}$. Standard treatment is either open appendectomy (OA) or laparoscopic appendectomy (LA), which can result in intra- and post-operative complications ranging from $2-23 \%^{3-5}$, with more than $3 \%$ of patients commonly readmitted with intestinal obstructions and postoperative adhesions ${ }^{6-8}$.

Recently, conservative treatment with antibiotics has been considered an alternative treatment option for uncomplicated appendicitis, including third generation cephalosporin (CEP), metronidazole (MET)/tinidazole, beta-lactam antibiotics, penicillin, and combinations thereof. The results from a recent network meta-analysis (NMA) ${ }^{9}$ suggest the use of antibiotics for treating uncomplicated appendicitis would result in about $12-32 \%$ lower treatment success at 1 year than appendectomy but about $23-86 \%$ fewer complications, which corresponded with findings from the previous meta-analysis ${ }^{10}$. In addition, this NMA also suggested that beta-lactam with/without penicillin was ranked first for treatment success and had the least complications compared to other antibiotic regimens. Furthermore, the most recent randomized control 
trial (RCT $)^{11}$ also found that the treatment success rate in 1 year was $65 \%$ or higher for both oral monotherapy of antibiotics and intravenous antibiotics followed by oral antibiotics.

Treatment of uncomplicated appendicitis should consider clinical outcomes as well as combination of health-related quality of life (HRQoL) cooperate with the cost as the cost-utility analysis (CUA). The majority of previous studies in adults have highlighted improved $\mathrm{HRQOL}$ associated with antibiotic treatments compared to LA ${ }^{12-14}$ in developed countries. Of them, only one ${ }^{15}$ considered a CUA with a hospital perspective reporting antibiotic treatment to be more cost effective than LA with cost reductions of 1,865 USD and 0.04 quality adjusted life years gained. Only a $\mathrm{RCT}^{16}$ in adults included a cost analysis with a societal perspective, reporting overall costs 1.6 times higher in LA compared to antibiotics. This study was continued to follow up at 7-year ${ }^{13}$ reporting no difference in long term HRQoL but lower satisfaction in patients who received antibiotics and later underwent appendectomy compared to patients with successful antibiotics or appendectomy outcomes. Most economic evidence has been generated from developed countries, evidence from developing countries is still lacking, particularly using CUA from a societal perspective. As such, this study used real practice data to assess HRQoL and CUA comparing individual antibiotics with appendectomy. This evidence will inform practitioners in treatment management for uncomplicated acute appendicitis.

\section{Material And Methods}

This prospective cohort study was conducted between November 2018 and October 2019 in the Department of Surgery, Faculty of Medicine, Ramathibodi Hospital, Bangkok, Thailand. This study had been approved and consent by the ethical committee of Ramathibodi Hospital (\#COA. MURA2019/1212). All adult patients who were diagnosed with uncomplicated appendicitis (proven by ultrasonography or computer tomography) were eligible if they received either antibiotics or appendectomy and were willing to participate following written informed consent. Patients later diagnosed as complicated appendicitis by ultrasonography or computer tomography were excluded. Patients were followed up their utilities for 1 month and complication/recurrence for 1 year.

CUA was performed to compare societal cost and utility scores between both interventions (antibiotic and appendectomy) using a decision tree model with one month time horizon and societal perspectives, see Fig. 1. One month time horizon was selected because most patient lose follow up from COVID pandemic. Cost benefit analysis was then performed for one-year time horizon to assess the net benefit by comparing cost of treatment with cost of complication or recurrence within one-year after discharge.

Interventions of interest included three antibiotics (beta-lactam, quinolone, and CET plus MET) and two operative modalities (OA and LA). LA was performed by single to three port incision and OA was performed using McBurney's point muscle-splitting incision immediately at the area of inflammation.

\section{Cost and utility score measurements}


Three main categories of direct medical cost data for medical services/resources and treatments for appendicitis patients between 2013 and 2017 was retrieved. All costs were adjusted to 2020 rates using an inflation rate from the national bank of Thailand. Costs were converted to USD (31.738 Baht/USD $)^{17}$ and classified into three categories: drug, equipment and staff. Direct non-medical and indirect costs were collected by patient interview at the following time points: before receiving intervention, discharge, 1-week and 1-month post discharge. HRQoL was measured by a European Quality of Life-5 Dimensions questionnaire at the time points indicated for each treatment with conversion to a Thai utility score reflecting their perception of their HRQoL. Scores ranged from 0 to 1 , with 0 meaning worse/death and 1 meaning better health status ${ }^{18}$. Patient's electronic medical record was reviewed for any complication or recurrence occurred within one-year after discharge.

\section{Transition probability}

The transition probability of a decision tree model demonstrates the effect size for each outcome of interest and the corresponding confidence intervals $(95 \% \mathrm{Cl})$. For antibiotic treatments, probabilities of transition to success (complete response without recurrence), failure (did not complete response during admission), and recurrence (repeated occurrence of appendicitis within one month), were applied based on the NMA effect size and $95 \% \mathrm{Cl}^{9}$, while for operative treatments, transition probabilities of success (complete operation and confirmed pathology as acute appendicitis), complication-free (success of the initial operation with

no postoperative complications, adverse events, or operative failure occurring) and complications (major complications of operative treatment including: wound infection, wound hematoma, wound dehiscence, intra-abdominal abscess and other complications within one month) ${ }^{10}$ were applied according to the effect size and $95 \% \mathrm{Cl}$ from an umbrella review ${ }^{19}$.

\section{Statistical analysis}

Data were described using mean ( \pm standard deviation) and frequency $(\%)$ for continuous and categorical data, respectively. The utility scores following treatment were compared between interventions using a treatment effect model with inverse-probability-weighted regression adjustment (IPWRA) ${ }^{20}$. Two equations were constructed. Firstly, a treatment model was constructed based on regressing interventions on covariables (i.e., age, BMI, nausea, neutrophil/lymphocyte ratio, utility score at baseline, and time) using a multi-logit link function. A propensity score was estimated as a weight in step 2 . Secondly, the outcome model constructed was based on regressing the utility score for interventions with IPWRA with adjustment for co-variables (i.e., age, BMI, nausea, neutrophil/lymphocyte ratio, and utility score at baseline). Potential outcome mean (POM) and average treatment effect (ATE) were estimated for each intervention.

For CUA, an incremental cost-effectiveness ratio (ICER) was calculated by dividing the incremental total admission cost by the incremental utility score of any paired intervention comparisons ${ }^{18}$. A one-way sensitivity analysis of the ICER was depicted in a Tornado diagram with variable parameter and $\mathrm{Cl}$ estimates. Probabilistic sensitivity analysis to account for the uncertainty in the CUA model considered 1,000 simulations by Monte-Carlo methods based on beta and gamma distributions for incremental utilities 
and costs, respectively. As a result, the model presentation was estimated in the CE scatter plot and was evaluated against the ceiling ratio or willingness to pay (WTP) in a CE acceptability curve. For cost benefit analysis, a net benefit was calculated by subtracting incremental benefit from avoided cost of any recurrence versus complication within 1 year with the incremental overall admission cost of antibiotics versus surgery; a net benefit is higher than 0 refers to lower benefit of intervention than comparator. Data were analyzed in STATA version 16, and all simulations were performed by TreeAge ${ }^{\circledR}$ Pro version $2020^{21}$.

\section{Results}

A total of 226 patients with uncomplicated acute appendicitis were included in the study. Among them, 138 (61\%), 8 (3.5\%), 18 (7.9\%), $13(5.7 \%)$, and 49 (21.6\%) patients received OA, LA, beta-lactam, quinolone, and CEP+MET, respectively. Mean ( \pm standard deviation) utility scores at baseline between the five groups were not significantly different with values of $0.4(0.2), 0.4(0.1), 0.4(0.2), 0.5(0.1)$, and $0.4(0.1)$, see Table 1 . The mean age and BMI were also not significantly different between groups, although diabetes mellitus, nausea, vomiting, and median neutrophil/lymphocyte ratio were statistically different (P-value: 0.016, 0.011, 0.007, and $<0.001$ respectively). Occurrence of complications in OA and LA ranged from $17 \%$ to $56 \%$ and $20 \%$ to $44 \%$, respectively, see Table 4. All patients were available for interview at the 1 month follow-up.

\section{Comparison of utility scores}

A counterfactual approach was applied to assess the effects of the five treatments on utility scores. The treatment model was constructed to balance factors associated with treatment allocations, see supplementary document. Three factors (i.e., nausea, neutrophil/lymphocyte ratio, and baseline utility score) were significantly associated with treatment allocation between intervention groups.

The outcome model was generated from the IPWRA estimates from the treatment model. The POMs for the utility scores following treatment during hospitalization were $0.91,0.89,0.91,0.30$ and 0.03 for beta-lactam, quinolone, $C E P+M E T$, LA and OA respectively. As a result, the ATEs were significantly higher for the three antibiotics and LA relative to $O A$ with values of $0.88(0.83,0.93), 0.86(0.78,0.93), 0.87(0.82,0.92), 0.26$ $(0.11,0.42)$ respectively, see Table 2.

The utility scores at one month follow-up were much improved for all interventions, i.e., 1.00, 1.00, 0.99, 0.93, and 0.98 for beta-lactam, quinolone, CEP+MET, LA, and OA, respectively, see Table 2 . The ATEs $(95 \% \mathrm{Cl})$ were not significant for the interventions with the exception of beta-lactam with values of $0.03(0.01,0.05)$, $0.03(-0.004,0.06)$, and $0.01(-0.02,0.04),-0.05(-0.10,0.01)$, respectively.

CUA

The CUA compared cost and utility of antibiotic and operative treatments for uncomplicated acute appendicitis with a societal perspective. Direct medical costs associated with appendectomy drugs and equipment were higher than with antibiotic treatments. Furthermore, direct non-medical and indirect costs associated with operative treatments were also higher compared to antibiotics, see Table 3. 
Overall means for utility scores and transition probabilities for one month post antibiotic and operative treatments were estimated and stratified by complication/recurrence, see Table 4. For OA and LA, the utility scores ranged between $0.50-0.71$ and $0.66-0.94$ with and without wound complications. Taking into account recurrence of appendicitis, the utility scores for antibiotics with were $0.60,0.74$, and 0.66 for betalactam, CEP+MET, and quinolone. Utility scores for all interventions were higher in the absence of complications or recurrence of appendicitis, i.e., $0.58,0.66,0.96,0.97$, and 0.98 , respectively for beta-lactam, quinolone, CEP+MET, LA and OA.

Data from the decision tree model yielded ratios of cost to effectiveness of $773.62,654.44,653.32,5,373.86$, and 6,339.32 USD per utility gained for beta-lactam, quinolone, CEP+MET, LA, and OA, respectively, see Table 5. Comparisons between overall antibiotic and appendectomy $(O A+L A)$ resulted in cost-savings with an ICER of $-9,585.66$ USD per utility gained. Among the antibiotics investigated, the ICERs for beta-lactam and quinolone relative to CEP+MET were $-27,914.66$ and 1,164.23 USD respectively per utility gained. The ICER for LA relative to OA was 2,536.06 USD per utility gained. Overall, at 1 month post treatment, beta-lactam was most cost-effective in comparison to the other antibiotics and OA was more cost-effective compared to LA.

A sensitivity analysis included a Tornado diagram and the results are presented in Figure 2 . The Tornado diagram ordered the most sensitive interventions as the utility of LA with wound complications (ICER = $-15,000.00$ to $-6,500.00)$, LA with other complications $(-12,500.00$ to $-7,500.00)$, transition probability of LA with other complications $(-11,250.00,-8000.00)$, utility of quinolone with recurrence $(-11,250.00,-8500.00)$, and utility of quinolone without recurrence $(-11,750.00,-9,500.00)$.

Incremental cost and effectiveness scatter plots were based on 1,000 Monte-Carlo simulations, see Figure 3. Each plot was divided into 4 areas, i.e., the southeast quadrant (cost-saving, i.e., more effectiveness at less cost), northwest quadrant (i.e., more cost and less effectiveness), northeast quadrant (i.e., more cost and more effectiveness) and southwest (i.e., less cost and less effectiveness); the last two quadrants are required for the comparison of WTP thresholds. Antibiotics offered greater cost-savings in comparison to appendectomy, see Figure 3A. For the 2 different types of appendectomy, the plots were scattered in all quadrants but most of them were in the southeast quadrant, i.e. LA might be more effective than OA, see Figure 3B. Among antibiotic treatments, most values were centrally scattered for all comparisons (see Figure 3C-3E), suggesting the cost-effectiveness of antibiotics as a treatment option might be dependent on WTP.

Cost-effectiveness acceptability curves (CEAC) illustrate the percentage cost-effectiveness (y-axis) in relation to a given WTP (x-axis), see Figure 4. Antibiotics were more cost-effective for all WTP levels compared to overall appendectomy, see Figure 4A. Likewise, LA was more cost-effective in comparison to OA for all WTP thresholds (Figure 4B). For antibiotic comparisons, quinolone was the most cost-effective treatment, followed by beta-lactam at WTP thresholds in excess of 40,000 USD per utility gained.

\section{Cost benefit analysis}

As described in Table 3, the overall incremental cost (i.e., direct medical, non-medical and indirect costs) of antibiotic treatments versus surgery was $-3,265.26$ USD. During 1 year follow up, there were $4(5.0 \%)$ out of 
80 patients in antibiotic groups had recurrence of appendicitis whereas $6(4.1 \%)$ out of 146 patients in surgery had complications. The average treatment cost per case for recurrence and complication were 536.28 USD and 282.53 USD, respectively. As a result, the incremental cost of recurrence versus complication was 15.23 USD (i.e., $(536.28 \times 5.0 \%)-(282.53 \times 4.1 \%))$, which resulted in the net benefit of $3,250.08$ USD. This could be interpreted that antibiotics had lower benefit, or in other words, surgery had better benefit than antibiotics.

\section{Discussion}

This cohort study compared utility scores and CUA of three antibiotics (beta-lactam, quinolone, and CEP + MET) and two appendectomy modalities (LA and OA) in uncomplicated acute appendicitis. The ATEs for antibiotics (beta-lactam, quinolone, and CEP + MET) and appendectomy (OA and LA) during hospitalization and one month after discharge were considered for each outcome of interest (success, complications and recurrence). The ICER identified CEP + MET with the lowest cost at 653.32 USD, followed by quinolone (654.44), beta-lactam (773.62), LA (5,373.86), and OA (6,339.32), for a single utility gain one-month after treatment. CE ratios with time horizon of one month, estimated that operative treatment cost 8-10 times more compared to any antibiotic use.

HRQoL utility scores were significantly higher for beta-lactam, quinolone, and CEP + MET compared to either LA/OA following treatment during hospitalization beta-lactam. However, overall utility scores at one month post treatment were much improved for all interventions, with only beta-lactam demonstrating a significantly higher utility scores approximating $0.03(0.01,0.05)$ compared to OA beta-lactam. This difference might not be clinically significant as for the previous study also found antibiotics were noninferior to appendectomy with the difference of 30-day utility score of $0.01(-0.001,0.03)^{22}$. However, previous comparisons of antibiotics with LA reported a bit higher 1-year HRQoL values associated with antibiotic treatments compared to $L A\left(0.872\right.$ versus 0.802 in adults) ${ }^{15}$. In addition, a recent $R C T^{13}$ showed similar HRQoL at 7 year long term follow-up for antibiotic and operative treatments with a median health index value $(95 \% \mathrm{Cl})$ of $1.0(0.86,1.0)$, with patient satisfaction indicating those who received operative treatments were more satisfied than patients who had received antibiotic treatment $(68 \%$ versus $53 \%$, respectively). Previous HRQoL evidence over equivalent timeframes have shown similar findings between antibiotics and appendectomy and as such, the level of variation in the utility scores may be time dependent.

Antibiotic treatment of uncomplicated acute appendicitis patients lead to short-term cost-savings compared to appendectomy, with a net patient utility gain for a lower cost, i.e. ICER of $-9,587.86$ USD per utility gained. Antibiotic treatment comparisons identified CEP + MET to be superior to beta-lactam with lower cost and a higher utility gain. Nevertheless, quinolones may be more cost-effective if the threshold exceeds 1,164.23 USD per utility gained. Likewise, OA is more cost effective than LA when the ICER threshold exceeds 2,536.06 USD per utility gained.

Our CUA showed that antibiotic treatment options presented cost-savings compared to operative treatment options with ICER of $-9,585.66$ USD per utility score unit gain. These findings support previously reported 
cost effectiveness of antibiotic use over operative treatment with ICER ranging between 1,865-4,271 USD per QALY for antibiotics treatment compared to 172,600 - 427,100 USD per QALY for operative treatments 12,15 . The CE ratio for operative treatments in our study was higher compared to that for antibiotic treatments, similar to previous RCT, although the latter had not also considered utility ${ }^{16}$.

Our previous $\mathrm{NMA}^{9}$ suggested that all antibiotics were about $12-32 \%$ lower in success rates when compared with appendectomy, which was corresponded with the previous meta-analysis ${ }^{10}$. Among comparison of $\mathrm{LA}$ and $\mathrm{OA}{ }^{19}$, from our umbrella review of 10 systematic reviews and meta-analyses suggested that LA had significantly lower risk of infection from 0.47 to 0.67 but showed higher risk of intraabdominal abscess about 1.20 times than OA. Although, the benefit of LA seem to be better when compared with the risks. The recent study also showed that a per-oral antibiotic treated in out-patients might be sufficient relative to intravenous followed by oral antibiotics treated in-patients with acute uncomplicated appendicitis.

The issue of antibiotic resistance should also be considered as for the growth of Clostridium difficile in intestinal flora among antibiotic users ${ }^{23}$. Used of broad-spectrum antibiotics is more likely to develop flora infections when compare to penicillin regimens combinations ${ }^{24}$. Long term antibiotic resistance among different types of antibiotics should be further studied to support this.

Our study had several strengths. We conducted a cohort alongside CUA of adults with acute uncomplicated appendicitis. We compared utility scores using a counter-factual approach by treatment effect model. Our data was representative of real clinical practice to emulate a randomized-controlled trial ${ }^{25}$, using the IPWRA method. All costs, including direct medical and non-medical costs, and indirect costs with societal perspectives, were appropriately considered. Standard direct costs from the Ramathibodi Hospital were used which are generalizable to similar healthcare settings and economic models.

Our study also had several limitations. HRQoL was assessed in only short-term, and thus may be invalid estimations, i.e., either under and over estimations. Although we had considered and accounted the cost of recurrences in antibiotics and complications in surgery at one years, we could not collected the HRQoL from the patients because of the pandemic of COVID-19. We could only assess the cost benefit analysis, which was not the full scheme of HRQoL for decision making. In addition, the sample size for each antibiotic and LA/OA against individual outcomes (i.e., recurrence, wound complication) was small, therefore, analysis of individual antibiotic and operation approach could not be done, and also limiting the certainty associated with the HRQoL for each branch of the decision tree. Furthermore, LA was more commonly used in developed countries, while it was still very much less applied relative to OA in our and other settings in developing countries, which was due to the cost of implementation and maintenance, and the lack of trained surgeons ${ }^{26} 27$. Therefore, our findings were less precise particularly the LA, and may be less generalizable to countries where LA was commonly applied.

In summary, our findings suggest that antibiotic treatments may represent a higher utility score and CE than appendectomy. Costs associated with antibiotic treatments may be as much as 8 to 10 times lower than appendectomy. Therefore, antibiotics should be considered as a treatment option for patients if surgery is 
contraindicated, or patients prefer a non-surgical alternative. However, the potential post treatment consequences particularly for appendicitis recurrence and post-operative complications, should be carefully considered in a balanced risk benefit assessment.

\section{Conclusions}

Antibiotics may represent a more cost effective treatment option for the management of acute uncomplicated adult appendicitis. Therefore, antibiotic treatment might be offered as an alternative to patients with contra-indications for surgery, to those who prefer not to undergo surgery or to patients who are more likely to develop post-surgical complications. However, this recommendation is based on shortterm evaluation, further long-term study is required.

\section{Abbreviations}

\begin{tabular}{|ll|}
\hline ATE & Average treatment effect \\
\hline BMI & Body mass index \\
\hline CEAC & Cost-effectiveness acceptability curves \\
\hline CE & Cost-effectiveness \\
\hline CEP & Cephalosporin \\
\hline CUA & Cost utility analysis \\
\hline HRQoL & Health-related quality of life \\
\hline ICER & Incremental cost-effectiveness ratio \\
\hline IPWRA & Inverse-probability-weighted regression adjustment \\
\hline LA & Laparoscopic appendectomies \\
\hline MET & Metronidazole \\
\hline NMA & Network meta-analysis \\
\hline OA & Open appendectomy \\
\hline POM & Potential outcome means \\
\hline RCT & Randomized controlled trials \\
\hline WTP & willingness to pay \\
\hline
\end{tabular}

\section{Declarations}

Ethics approval and consent to participate

Ethics approval and consent to participate 
This study had been approved and consent by the ethical committee of Ramathibodi Hospital (\#COA. MURA2019/1212).

Consent for publication

Informed consent was obtained from all participating patients.

\section{Availability of data and materials}

The datasets used are available from the corresponding author upon reasonable request.

\section{Competing interests}

This study was conducted without any potential conflict of interest.

\section{Funding}

None.

\section{Authors' contributions}

NP: Study concept and design, acquisition of data, analysis and interpretation of data, drafting of the manuscript, and critical revision of the manuscript.

OP: Study concept and design, analysis and interpretation of data, and critical revision of the manuscript. CW: Technical and material support, and critical revision of the manuscript.

SR: Acquisition of data, analysis and interpretation of data.

GM: Critical revision of the manuscript.

JA: Critical revision of the manuscript.

AT: Concept and design, analysis and interpretation of data, drafting of the manuscript, and critical revision of the manuscript.

All authors read and approved the final manuscript.

\section{Acknowledgements}

Not applicable.

\section{References}

1. Di Saverio S, Sibilio A, Giorgini E, Biscardi A, Villani S, Coccolini F, Smerieri N, Pisano M, Ansaloni L, Sartelli M, et al. The NOTA Study (Non Operative Treatment for Acute Appendicitis): prospective study 
on the efficacy and safety of antibiotics (amoxicillin and clavulanic acid) for treating patients with right lower quadrant abdominal pain and long-term follow-up of conservatively treated suspected appendicitis. Ann Surg. 2014;260(1):109-117.

2. Salminen P, Paajanen H, Rautio T, Nordstrom P, Aarnio M, Rantanen T, Tuominen R, Hurme S, Virtanen J, Mecklin JP, et al. Antibiotic Therapy vs Appendectomy for Treatment of Uncomplicated Acute Appendicitis: The APPAC Randomized Clinical Trial. Jama. 2015;313(23):2340-2348.

3. Emil S, Duong S. Antibiotic therapy and interval appendectomy for perforated appendicitis in children: a selective approach. Am Surg. 2007;73(9):917-922.

4. Malik AA, Bari SU. Conservative management of acute appendicitis. Journal of gastrointestinal surgery : official journal of the Society for Surgery of the Alimentary Tract. 2009;13(5):966-970.

5. Omundsen M, Dennett E. Delay to appendicectomy and associated morbidity: a retrospective review. ANZ J Surg. 2006;76(3):153-155.

6. Leung TT, Dixon E, Gill M. Bowel obstruction following appendectomy what is the true incidence? Ann Surg. 2009;250:51-53.

7. Parker MC, Ellis H, Moran BJ. Postoperative adhesions: ten-year follw-up of 12,584 patients undergoing lower abdominal surgery. Dis Colon Rectum. 2001;44:822-829.

8. Vons C, Barry C, Maitre S, Pautrat K, Leconte M, Costaglioli B, Karoui M, Alves A, Dousset B, Valleur P, et al. Amoxicillin plus clavulanic acid versus appendicectomy for treatment of acute uncomplicated appendicitis: an open-label, non-inferiority, randomised controlled trial. Lancet. 2011;377(9777):15731579.

9. Poprom N, Numthavaj P, Wilasrusmee C, Rattanasiri S, Attia J, McEvoy M, Thakkinstian A. The efficacy of antibiotic treatment versus surgical treatment of uncomplicated acute appendicitis: Systematic review and network meta-analysis of randomized controlled trial. Am J Surg. 2019;218(1):192-200.

10. Harnoss JC, Zelienka I, Probst P, Grummich K, Muller-Lantzsch C, Harnoss JM, Ulrich A, Buchler MW, Diener MK. Antibiotics Versus Surgical Therapy for Uncomplicated Appendicitis: Systematic Review and Meta-analysis of Controlled Trials (PROSPERO 2015: CRD42015016882). Ann Surg. 2016.

11. Sippola S, Haijanen J, Grönroos J, Rautio T, Nordström P, Rantanen T, Pinta T, Ilves I, Mattila A, Rintala J, et al. Effect of Oral Moxifloxacin vs Intravenous Ertapenem Plus Oral Levofloxacin for Treatment of Uncomplicated Acute Appendicitis: The APPAC II Randomized Clinical Trial. Jama. 2021;325(4):353362.

12. Sceats LA, Ku S, Coughran A, Barnes B, Grimm E, Muffly M, Spain DA, Kin C, Owens DK, GoldhaberFiebert JD. Operative Versus Nonoperative Management of Appendicitis: A Long-Term Cost Effectiveness Analysis. MDM policy \& practice. 2019;4(2):2381468319866448.

13. Sippola S, Haijanen J, Viinikainen L, Gronroos J, Paajanen H, Rautio T, Nordstrom P, Aarnio M, Rantanen T, Hurme S, et al. Quality of Life and Patient Satisfaction at 7-Year Follow-up of Antibiotic Therapy vs Appendectomy for Uncomplicated Acute Appendicitis: A Secondary Analysis of a Randomized Clinical Trial. JAMA Surg. 2020.

14. Turhan AN, Kapan S, Kütükçü E, Yiğitbaş H, Hatipoğlu S, Aygün E. Comparison of operative and non operative management of acute appendicitis. Ulusal Travma ve Acil Cerrahi Dergisi. 2009;15(5):459- 
462.

15. Wu JX, Dawes AJ, Sacks GD, Brunicardi FC, Keeler EB. Cost effectiveness of nonoperative management versus laparoscopic appendectomy for acute uncomplicated appendicitis. Surgery. 2015;158(3):712721.

16. Sippola S, Grönroos J, Tuominen R, Paajanen H, Rautio T, Nordström P, Aarnio M, Rantanen T, Hurme S, Salminen P. Economic evaluation of antibiotic therapy versus appendicectomy for the treatment of uncomplicated acute appendicitis from the APPAC randomized clinical trial. The British journal of surgery. 2017;104(10):1355-1361.

17. Bank of Thailand. Foreign Exchange Rates. Bangkok: Bank of Thailand; 24 Jul 20202020.

18. Pattanaphesaj J, Thavorncharoensap M, Ramos-Goñi JM, Tongsiri S, Ingsrisawang L, Teerawattananon Y. The EQ-5D-5L Valuation study in Thailand. Expert Rev Pharmacoecon Outcomes Res. 2018;18(5):551-558.

19. Poprom N, Wilasrusmee C, Attia J, McEvoy M, Thakkinstian A, Rattanasiri S. Comparison of postoperative complications between open and laparoscopic appendectomy: An umbrella review of systematic reviews and meta-analyses. The journal of trauma and acute care surgery. 2020;89(4):813820.

20. Stata Statistical Software: Release 16 [computer program]. College Station, TX: StataCorp LLC; 2019.

21. TreeAge Software [computer program]. Williamstown, MA: TreeAge Software, Inc; 2020.

22. Flum DR, Davidson GH, Monsell SE, Shapiro NI, Odom SR, Sanchez SE, Drake FT, Fischkoff K, Johnson J, Patton JH, et al. A Randomized Trial Comparing Antibiotics with Appendectomy for Appendicitis. The New England journal of medicine. 2020;383(20):1907-1919.

23. Huston JM, Kao LS, Chang PK, Sanders JM, Buckman S, Adams CA, Cocanour CS, Parli SE, Grabowski J, Diaz J, et al. Antibiotics vs. Appendectomy for Acute Uncomplicated Appendicitis in Adults: Review of the Evidence and Future Directions. Surg Infect (Larchmt). 2017;18(5):527-535.

24. Slimings C, Riley TV. Antibiotics and hospital-acquired Clostridium difficile infection: update of systematic review and meta-analysis. The Journal of antimicrobial chemotherapy. 2014;69(4):881-891.

25. Desai RJ, Franklin JM. Alternative approaches for confounding adjustment in observational studies using weighting based on the propensity score: a primer for practitioners. Bmj. 2019;367:15657.

26. Pizzol D, Trott M, Grabovac I, Antunes M, Colangelo AC, Ippoliti S, Ilie CP, Carrie A, Veronese N, Smith L. Laparoscopy in Low-Income Countries: 10-Year Experience and Systematic Literature Review. Int J Environ Res Public Health. 2021;18(11).

27. Alfa-Wali M, Osaghae S. Practice, training and safety of laparoscopic surgery in low and middle-income countries. World journal of gastrointestinal surgery. 2017;9(1):13-18.

\section{Tables}

Table 1. Baseline patient characteristics 
Characteristics

$\begin{array}{lllll}O A & \text { LA } & \begin{array}{l}\text { Bata- } \\ \text { lactam }\end{array} & \text { Quinolone } & \text { CEP+M } \\ (n=138) & (n=8) & (n=18) & & (n=49)\end{array}$

Baseline information

Age, years, mean (SD)

$42(17)$

$46(25)$

$42(20)$

$41(13)$

$38(18)$

0.277

Sex, number (\%)

Male, number (\%)

$58(42.0) \quad 3(37.5) \quad 5(27.7)$

$2(15.3)$

$21(42.8) \quad 0.307$

Female, number (\%)

$80(57.9)$

$5(62.5)$

$13(72.2) \quad 11(84.6)$

$28(57.1)$

Weight, kg, mean (SD)

$61.1(12.3)$

66.6

61.1

(12.1)

61.0

(11.8)

62.6

(13.0)

Height, cm, mean (SD)

$162.2(8.6)$

161.1

(10.10)

$161.0 \quad 160.4$

(7.7)

(5.2)

BMI, kg/m², mean (SD)

$23.1(3.9)$

$25.5(4.1)$

23.5

(4.3)

$23.6(3.9) \quad 23.4(4.5) \quad 0.838$

Diabetes, number (\%)

$6(4.3)$

$3(37.5)$

0

0

$1(2.0)$

$0.016 \ddagger$

Hypertension, number

(\%)

$17(12.3)$

$2(25.0)$

$2(11.1)$

0

$3(6.1)$

$0.290 \ddagger$

Sign \& Symptom

Nausea, number (\%)

71 (51.4)

$2(25.0)$

3 (16.6)

$3(23.0)$

$18(36.7)$

0.011

Vomiting, number (\%)

$53(38.4)$

$3(16.6) \quad 2(15.3)$

9 (18.3)

$0.007 \neq$

Sign \& Symptom

\begin{tabular}{|c|c|c|c|c|c|c|}
\hline Anorexia, number (\%) & $11(7.9)$ & 0 & $1(5.5)$ & 0 & $3(6.1)$ & $0.978 \ddagger$ \\
\hline $\begin{array}{l}\text { Onset of pain, h, median } \\
\text { (IQR) }\end{array}$ & $24(10,24)$ & $\begin{array}{l}24(17, \\
48)\end{array}$ & $\begin{array}{l}24(6 \\
72)\end{array}$ & $24(6,24)$ & $24(8,48)$ & $1.000 \dagger$ \\
\hline $\begin{array}{l}\text { Body temperature, }{ }^{\circ} \mathrm{C} \\
\text { mean (SD) }\end{array}$ & $37.4(0.8)$ & $37.2(0.8)$ & $\begin{array}{l}37.1 \\
(0.7)\end{array}$ & $37.8(1.0)$ & $37.0(0.7)$ & 0.527 \\
\hline \multicolumn{7}{|l|}{ Laboratory } \\
\hline $\begin{array}{l}\text { White blood cell count, } \\
\text { cell/ } \mathrm{mm}^{3} \text {, } \\
\text { mean (SD) }\end{array}$ & $\begin{array}{l}13514.3 \\
(3916.8)\end{array}$ & $\begin{array}{l}9037.5 \\
(3396.2)\end{array}$ & $\begin{array}{l}11480.0 \\
(3924.4)\end{array}$ & $\begin{array}{l}11516.2 \\
(5360.9)\end{array}$ & $\begin{array}{l}11685.7 \\
(3959.5)\end{array}$ & 0.570 \\
\hline $\begin{array}{l}\text { Neutrophil/lymphocyte } \\
\text { ratio, } \\
\text { median (IQR) }\end{array}$ & $\begin{array}{l}8.82 \\
(4.8,15.3)\end{array}$ & $\begin{array}{l}3.0 \\
(2.2,3.9)\end{array}$ & $\begin{array}{l}3.7 \\
(1.9,6.4)\end{array}$ & $\begin{array}{l}5.6 \\
(2.9,9.1)\end{array}$ & $\begin{array}{l}4.6 \\
(3.0,5.6)\end{array}$ & $<0.001 \dagger$ \\
\hline
\end{tabular}

Utility score, mean (SD)

$0.4(0.2)$

$0.4(0.1) \quad 0.4(0.2)$

$0.5(0.1)$

$0.4(0.1)$

0.120 
CEP; Cephalosporin, LA; Laparoscopic appendectomy, MET; Metronidazole, OA; Open appendectomy. ‡ Fisher's exact test. †Quantile regression.

Table 2. POM and ATE estimates for utility index scores between treatment groups

\begin{tabular}{llllll} 
Time after receive treatments & Treatment & POM (SE) & ATEs & Lower limit & Upper limit \\
\hline Hospitalization & OA & $0.03(0.01)$ & REF & & \\
\cline { 2 - 6 } & LA & $0.30(0.07)$ & 0.26 & 0.11 & 0.42 \\
\hline & Beta-lactam & $0.91(0.01)$ & 0.88 & 0.83 & 0.93 \\
\hline Quinolone & $0.89(0.03)$ & 0.86 & 0.78 & 0.93 \\
\hline CEP+MET & $0.91(0.01)$ & 0.87 & 0.82 & 0.92 \\
\hline 1 month & OA & $0.98(0.01)$ & REF & & \\
\hline LA & $0.93(0.02)$ & -0.05 & -0.10 & 0.01 \\
\hline Beta-lactam & $1.00(0.01)$ & 0.03 & 0.01 & 0.05 \\
\hline Quinolone & $1.00(0.01)$ & 0.03 & -0.004 & 0.06 \\
\hline CEP+MET & $0.99(0.01)$ & 0.01 & -0.02 & 0.04
\end{tabular}

ATE; Average treatment effect, CEP; Cephalosporin, LA; Laparoscopic appendectomy, MET; Metronidazole, OA; Open appendectomy, POM; Potential outcome mean, REF; Reference.

Table 3. Associated costs of drugs, equipment and hospital staff for appendicitis patients stratified by intervention and complications 
Interventions Direct medical cost, USD (mean \pm SD)

Direct non- Indirect cost, medical cost, USD USD

Drug Equipment Staff

(mean $\pm S D)$

$(\operatorname{mean} \pm S D)$

$\begin{array}{llllll}\text { OA with } & 2469.7 \pm 3593.7 & 585.3 \pm 133.3 & 379.6 \pm 126.8 & 76.8 \pm 157.1 & 103.5 \pm 170.9\end{array}$ wound complications

OA with other complications

$2827.3 \pm 25317.5$

$591.9+210.5$

$347.4 \pm 10.2$

$78.7 \pm 0.1$

$31.5 \pm 0.1$

OA with no $2057.5 \pm 5760.9$

$724.4 \pm 14.6$

$314.1 \pm 28.2$

$187.6 \pm 141.7$

$5051.9 \pm 141.7$

complications

Overall OA

$7354.6+34672.2$

$1901.8 \pm 358.5$

$1041.1 \pm 165.3$

$178.8 \pm 146.8$

$4752.8 \pm 104.2$

LA with

wound $2997.1 \pm 582.9$

$338.2 \pm 266.7 \quad 652.3 \pm 11.1$

$83.8 \pm 0.1$

$31.5 \pm 0.1$

complications

LA with other complications

$2997.1 \pm 582.9$

$362.1 \pm 156.8$

$652.3 \pm 11.1$

$236.3 \pm 151.4$

$181.1 \pm 211.6$

LA with no
complications

Overall LA

$1498.5 \pm 956.9$

$1634.9 \pm 11.2$

$302.5 \pm 44.3$

$242.7 \pm 130.6$

$289.4 \pm 157.3$

Bata-lactam
with
recurrence

Bata-lactam

$4.9 \pm 0.1$

$2.2 \pm 0.06$

$352.8 \pm 0.2$

$204.71 \pm 324.3$

$184.7 \pm 161.2$

recurrence

CEP+MET

with

$52.1 \pm 91.0$

$180.5 \pm 5.8$

$897.4 \pm 20.8$

$82.0 \pm 55.6$

$126.0 \pm 55.6$

recurrence

CEP+MET

with no

$5.2 \pm 4.2$

$66.7 \pm 55.2$

$301.8 \pm 47.4$

$107.7 \pm 99.2$

$146.2 \pm 167.3$

recurrence

Quinolone

$89.3 \pm 0.1$

$177.2 \pm 0.3$

$881.2 \pm 0.1$

$74.9 \pm 27.6$

$31.5 \pm 0.1$

recurrence

Quinolone

with no

$149.3 \pm 290.1$

$26.0 \pm 6.7$

$256.7 \pm 30.5$

$100.1 \pm 54.6$

152.2 \pm 78.1

recurrence

Overall antibiotics

$632.6 \pm 300.3$

$700.9 \pm 72.4$

$3571.5 \pm 330.7$

$126.1 \pm 168.6$

$154.0 \pm 146.5$

CEP; Cephalosporin, LA; laparoscopic appendectomy, MET; Metronidazole, OA; open appendectomy. 
Table 4. Utility and transition probabilities used in the decision tree model

\begin{tabular}{lll} 
Utility & Mean \pm SD & Transition probability \\
\hline OA with wound complications & $0.50 \pm 0.06$ & 0.56 \\
\hline OA with other complications & $0.71 \pm 0.08$ & 0.17 \\
\hline OA with no complications & $0.58 \pm 0.01$ & 0.27 \\
\hline LA with wound complications & $0.73 \pm 0.01$ & 0.44 \\
\hline LA with other complications & $0.94 \pm 0.01$ & 0.20 \\
\hline LA with no complications & $0.66 \pm 0.05$ & 0.36 \\
\hline Bata-lactam with recurrence & $0.60 \pm 0.10$ & 0.18 \\
\hline Bata-lactam with no recurrence & $0.96 \pm 0.03$ & 0.82 \\
\hline CEP+MET with recurrence & $0.74 \pm 0.05$ & 0.29 \\
\hline CEP+MET with no recurrence & $0.97 \pm 0.02$ & 0.71 \\
\hline Quinolone with recurrence & $0.66 \pm 0.10$ & 0.23 \\
\hline Quinolone with no recurrence & $0.98 \pm 0.04$ & 0.77
\end{tabular}

CEP; Cephalosporin, LA; laparoscopic appendectomy, MET; Metronidazole, OA; open appendectomy.

Table 5. Cost, utility, CE ratio and ICER among and between intervention groups

\begin{tabular}{|c|c|c|c|c|c|}
\hline \multirow[t]{2}{*}{ Treatment } & \multirow{2}{*}{$\begin{array}{l}\text { Cost } \\
\text { (USD) }\end{array}$} & \multirow[t]{2}{*}{ Utility } & \multirow{2}{*}{$\begin{array}{l}\text { CE ratio } \\
\text { (USD/utility } \\
\text { gained) }\end{array}$} & \multicolumn{2}{|c|}{ ICER (USD/utility gained) } \\
\hline & & & & $\begin{array}{l}\text { Among } \\
\text { treatments }\end{array}$ & $\begin{array}{l}\text { Between } \\
\text { treatments }\end{array}$ \\
\hline Beta-lactam & 700.12 & 0.905 & 773.62 & $-27,914.66$ & \multirow[t]{3}{*}{$-9,585.66$} \\
\hline Quinolone & 596.88 & 0.912 & 654.44 & $1,164.23$ & \\
\hline CEP+MET & 593.21 & 0.908 & 653.32 & Referrence & \\
\hline Overall antibiotics & 808.45 & 0.910 & 888.41 & - & \\
\hline LA & $3,793.94$ & 0.706 & $5,373.86$ & $2,536.06$ & \\
\hline $\mathrm{OA}(\mathrm{RF})$ & $3,340.83$ & 0.527 & $6,339.32$ & Referrence & \\
\hline $\begin{array}{l}\text { Overall } \\
\text { appendectomy }\end{array}$ & $4,073.71$ & 0.630 & $6,466.21$ & - & \\
\hline
\end{tabular}


CEP; Cephalosporin, CE; cost-effectiveness, CU; cost utility ratio, ICER; incremental cost effectiveness ration, LA; Laparoscopic appendectomy, MET; Metronidazole, OA; Open appendectomy.

\section{Figures}

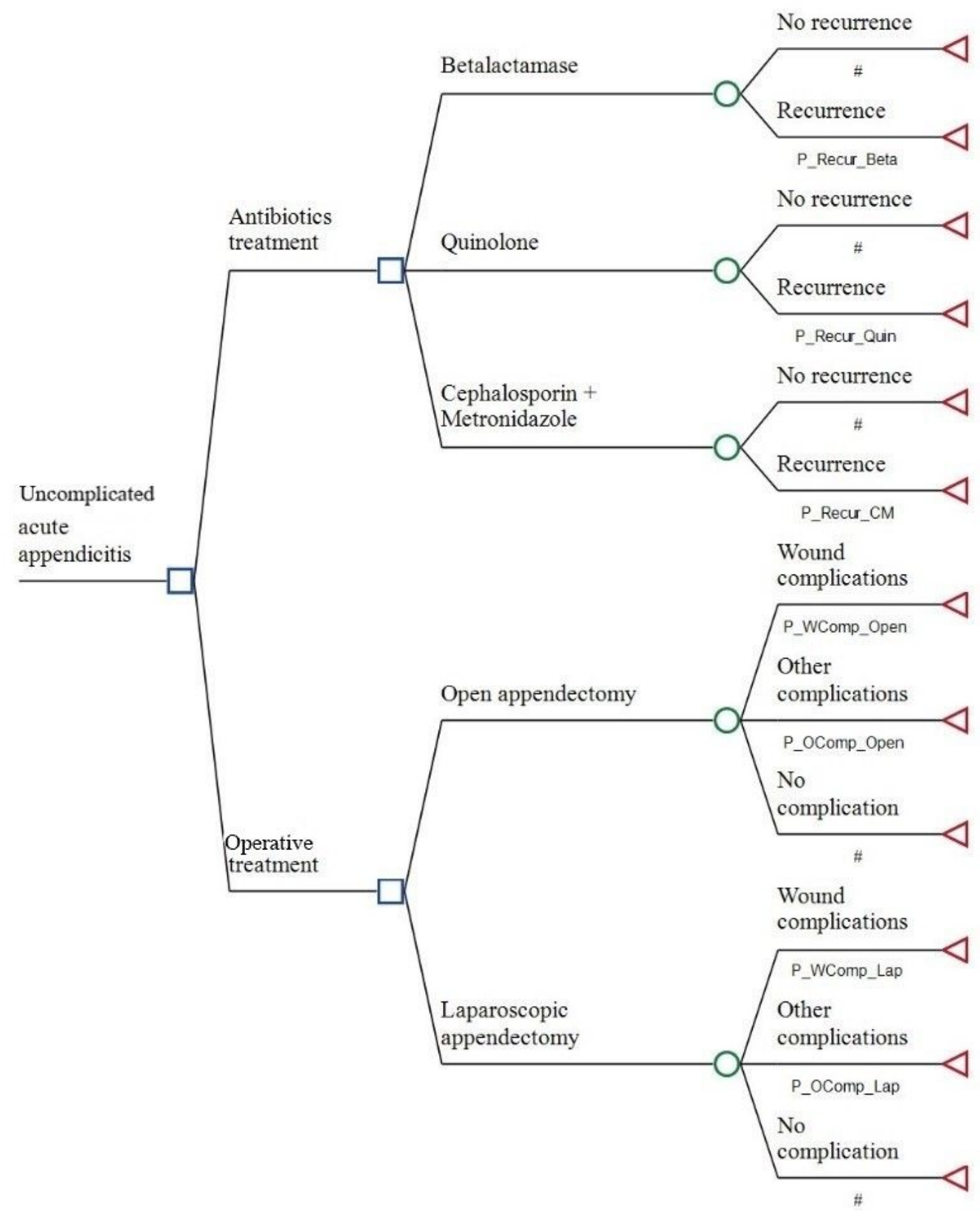

Figure 1 


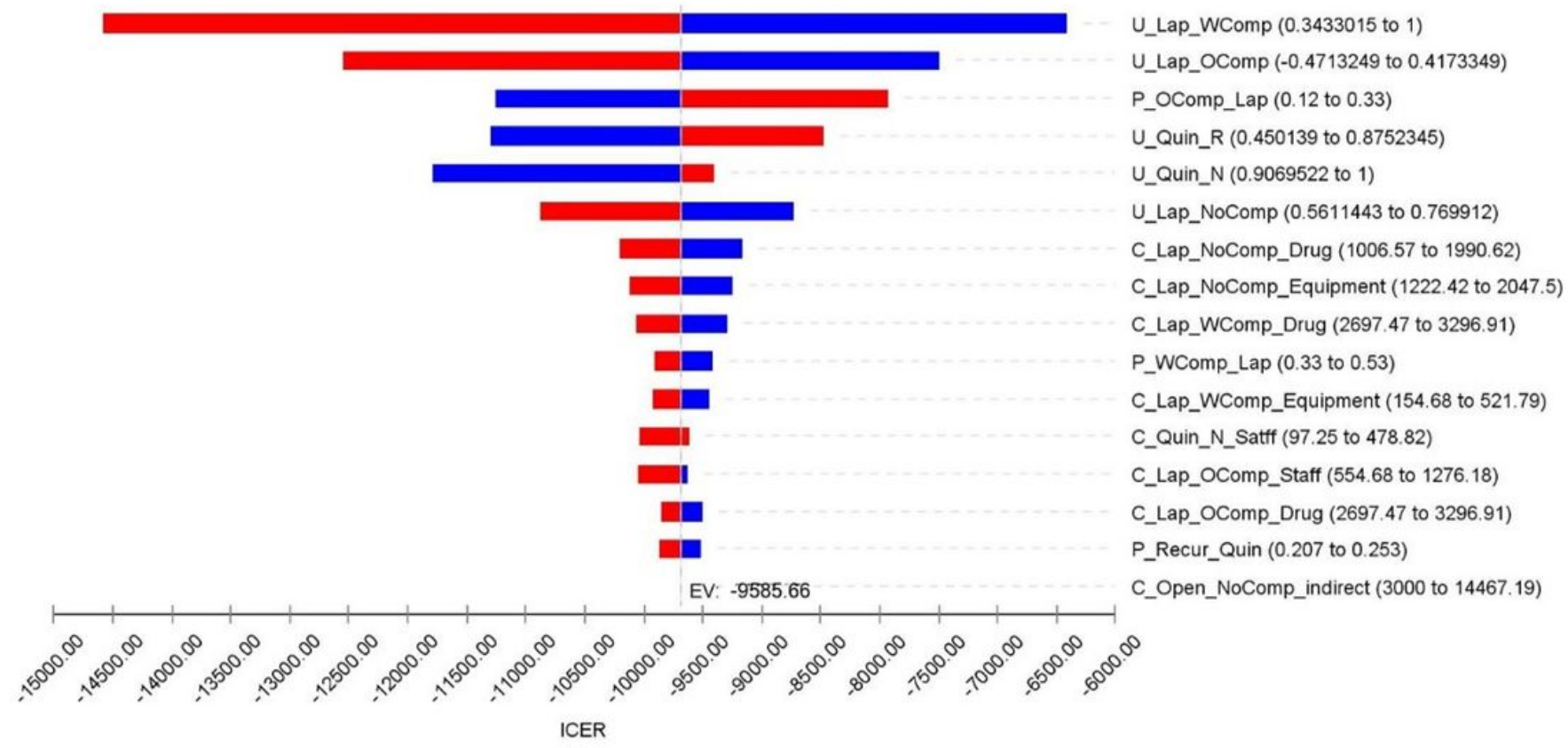

Cataredecompadug: Cost of drug of no complications in laparoscopic appendectomy,

SoLapaleComp Equipment: Cost of equipment of no complications in laparoscopic appendectomy,

Cortap WComp Drog; Cost of drug of wound complications in laparoscopic appendectomy,

Sertapowcemp Equipment; Cost of equipment of wound complications in laparoscopic appendectomy,

Solarolcomp Drug; Cost of drug of other complications in laparoscopic appendectomy,

Sertapeocemp_Staff; Cost of staff of other complications in laparoscopic appendectomy,

SoOpen NoComp_indirect; Indirect cost of no complications in open appendectomy,

Coluin_daStaff; Cost of no recurrences in quinolone treatment,

PalComp_Lap; Probability of other complications in laparoscopic appendectomy,

RoWComp_tap; Probability of wound complications in laparoscopic appendectomy,

P.Becur Quin; Probability of recurrence in quinolone treatment,

U.LaR_WCemp; Utility of wound complications in laparoscopic appendectomy,

U.Lap OComp; Utility of other complications in laparoscopic appendectomy,

U.LaRateComp; Utility of no complications in laparoscopic appendectomy,

U_Quin $R$; Utility of recurrence in quinolone treatment,

U.Quin_d: Utility of no recurrence in quinolone treatment.

\section{Figure 2}

\section{Tornado diagram -ICER, antibiotic versus operative treatments}


A) Antibiotics vs. Overall appendectomy

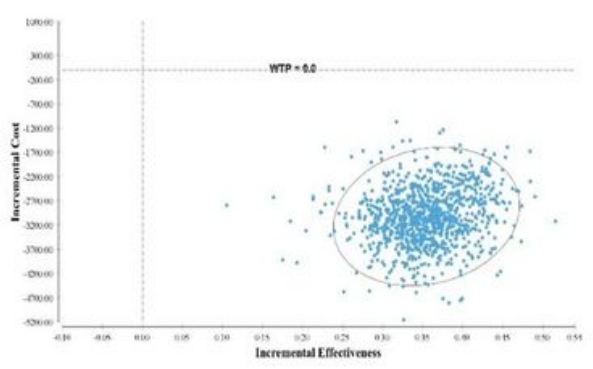

D) Quinolone vs. Cep+Met

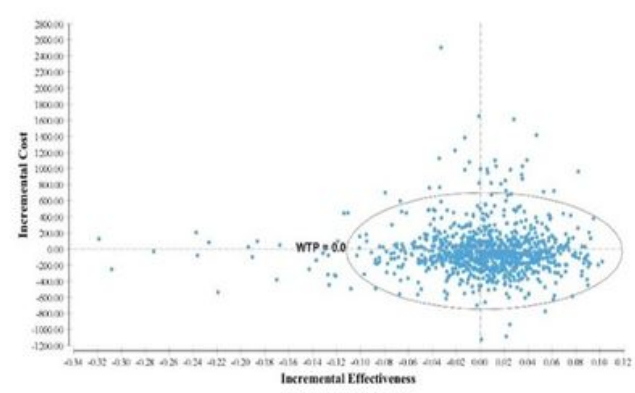

B) Laparoscopic vs. Open appendectomy

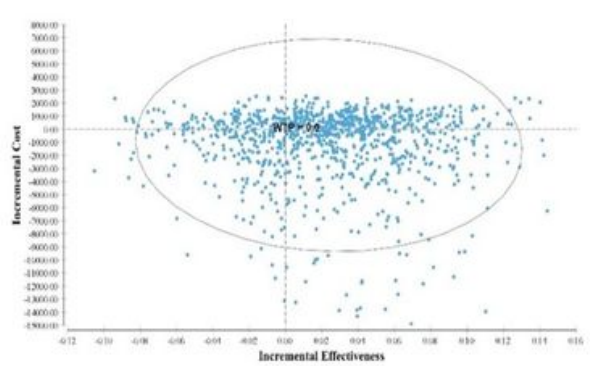

C) Beta-lactam vs. Cep+Met

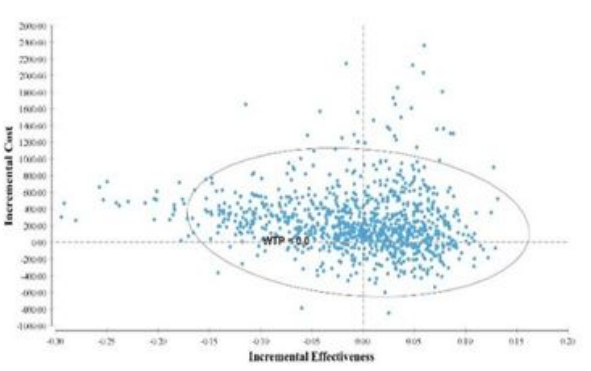

Cep; Cephalosporin, Met; Metronidazole, WTP; Willingness to pay.

\section{Figure 3}

Cost-effectiveness scatter plot between and among treatment groups 
A) Antibiotics vs. Overall appendectomy

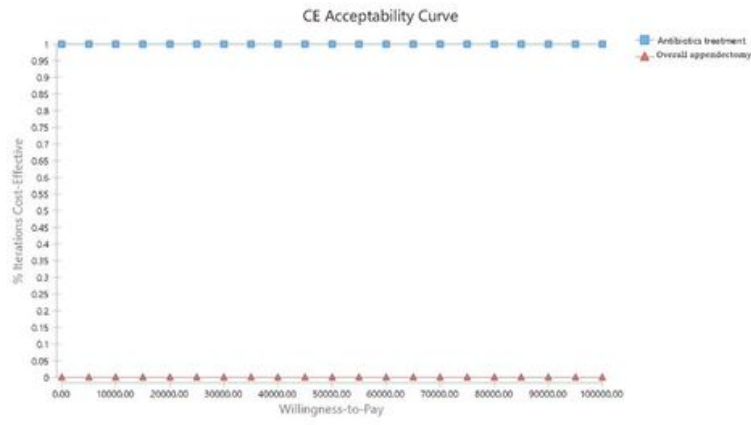

B) Laparoscopic vs. Open appendectomy

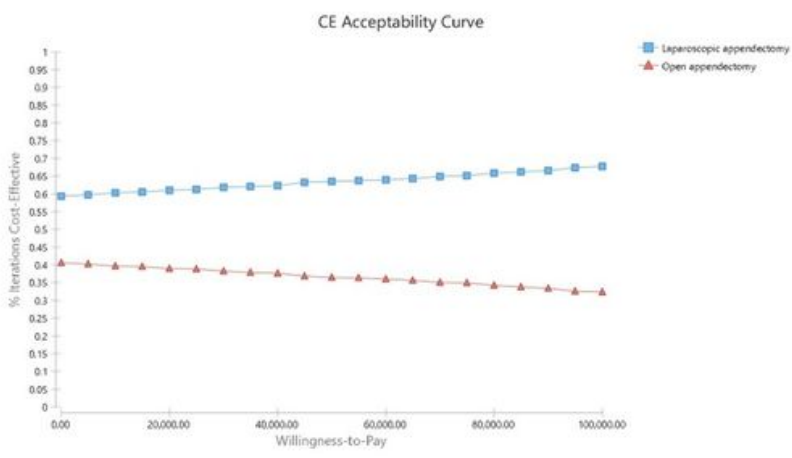

C) Beta-lactam vs. Cep+Met vs. Quinolone

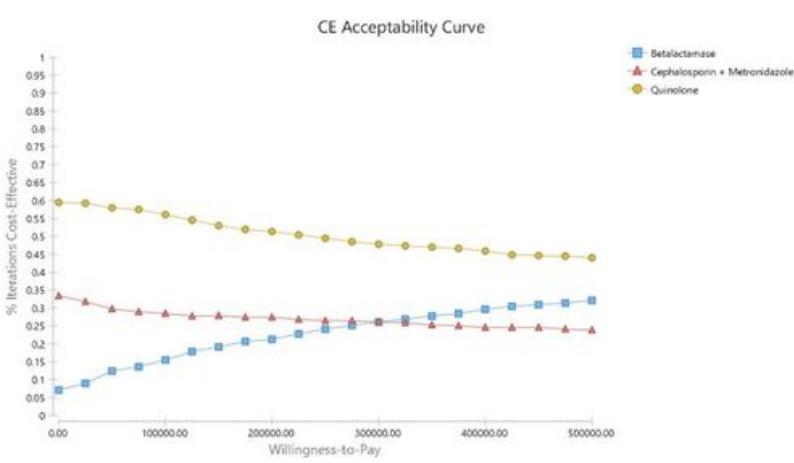

\section{Figure 4}

Cost-effectiveness acceptability curves between and among treatment groups 